\title{
Hash Functions And Graphs With Large Girths
}

\author{
G. Zémor \\ E.N.S.T. \\ Dept. Réseaux \\ 46 rue Barrault \\ 75634 Paris Cedex 13 \\ FRANCE
}

\section{ABSTRACT}

We propose and analyse an easily computable cryptographic hash function, for the purpose of signing long variable length texts, which is related to the construction of graphs with large girths.

\section{EXTENDED ABSTRACT}

We focus on the problem of devising an easily computable hash function, for integrity purposes. Such a function should be defined on the set of finite words over an alphabet, say $\{0,1\}^{*}$, and should take values on a small finite set ; its purpose is to provide long variable length texts (in practice of length several megabytes), with a "signature" (of a few hundred bits), without using a secret key. Computation should be fast, and finding a text with a given signature, or two texts with the same signature, (i.e. devising a forgery), difficult.

Several schemes have been proposed and analysed, one of which (see [God]), makes use of error-correcting codes, and has the attractive feature that modification of less than $d$ bits of text will necesserily yield a modification of the hashed value, where $d$ is the minimal distance of an appropriately chosen code. Unfortunately, such schemes are based on linear computations which are well known for their cryptographic weakness. We have tried to devise a hash function based on nonlinear computations which retains something of 
in the modular group $\Gamma=S L_{2}(Z) /\{1,-1\}$, we have $T^{2}=1$ and $(T S)^{3}=1$, furthermore it is well known that $\Gamma$ is isomorphic to the free product of a cyclic group generated by an $x$ of order 2 , and a cyclic group generated by a $y$ of order 3 , where $x$ corresponds to $T$ and $y$ corresponds to $T S$. From this it can easily be deduced that an equality of the form :

$$
X_{1} X_{2} \ldots X_{k} X_{h}^{\prime-1} \ldots X_{2}^{\prime-1} X_{1}^{\prime-1}=1
$$

with $X_{i}=A$ or $B$, and $X_{j}^{\prime}=A$ or $B$, cannot hold in $\Gamma$, unless $x_{1} \ldots x_{k}$ and $x_{1}^{\prime} \ldots x_{h}^{\prime}$ are identical strings.

Hence, if $x_{1} \ldots x_{k}$ and $x_{1}^{\prime} \ldots x_{h}^{\prime}$ are not identical :

$$
\left\|X_{1} X_{2} \ldots X_{k}-X_{1}^{\prime} X_{2}^{\prime} \ldots X_{h}^{\prime}\right\| \geq p
$$

where $\|M\|=\sup _{\xi \neq 0}\|M \xi\| /\|\xi\|$ and the norm of $\xi=\left(\begin{array}{l}\xi_{1} \\ \xi_{2}\end{array}\right)$ is $\|\xi\|=\sqrt{\xi_{1}^{2}+\xi_{2}^{2}}$.

By elementary linear algebra, $\|A\|=\sqrt{\left\|^{t} A A\right\|}=\sqrt{\lambda}$ where $\lambda$ is the largest eigenvalue of ${ }^{t} A A$. Hence $\|A\|=\|B\|=\phi=\frac{1+\sqrt{5}}{2} \approx 1.62$.

(3) implies

$$
\max \left(\left\|X_{1} X_{2} \ldots X_{k}\right\|,\left\|X_{1}^{\prime} X_{2}^{\prime} \ldots X_{h}^{\prime}\right\|\right) \geq p / 2
$$

and by the submultiplicativity of the norm of matrices

$$
\max (k, h) \geq \log _{\phi} \frac{p}{2}
$$

We have proved :

Proposition 1 If $k$ consecutive bits of text are replaced (nontrivially) by $h$ consecutive bits with $\max (k, h)<\log _{\phi} \frac{p}{2}$, then the signature is modified.

Compare this with an analogous result (obtained in very much the same way) stated in the language of graphs :

Proposition 2 The directed Cayley graph $\mathcal{C}\left(S L_{2}\left(F_{p}\right), A, B\right)$ has a girth (smallest circuitsize) $g \geq 2 \log _{\phi} \frac{p}{2}-1$.

The idea of using $S L_{2}\left(F_{p}\right)$ for constructing Cayley graphs with large girths originates in [Mar].

Hashed values range over $S L_{2}\left(F_{p}\right): A$ and $B$ are easily seen to generate $S L_{2}\left(F_{p}\right)$, but do texts of length a polynomial in $\log p$, yield arbitrary elements of $S L_{2}\left(F_{p}\right)$ as a hashed value ? (Clearly a necessary feature of an attractive hash function). This is indeed the case, i.e. we can prove : 
the features of the coding-based schemes ; for that purpose, we substituted the original tool, i.e. the minimum distance of a code, by the girth of a Cayley graph. This will be made clear shortly.

The scheme we propose is the following : to an arbitrary string of $\{0,1\}^{*}$ (the text) associate the string of $\{A, B\}^{*}$ obtained by substituting 0 for $A$ and 1 for $B$, then assign to $A$ and $B$ values of adequately chosen matrices of $S L_{2}(Z)$, those could be, for example :

$$
A=\left(\begin{array}{ll}
1 & 1 \\
0 & 1
\end{array}\right) \quad B=\left(\begin{array}{ll}
1 & 0 \\
1 & 1
\end{array}\right)
$$

then evaluate the product associated with the string of $A$ 's and $B$ 's in the group $S L_{2}\left(F_{p}\right)$ where $F_{p}$ is the field on $p$ elements, $p$ being a chosen large prime number (e.g. of 150 bits). The hashed value $S$ is the computed product (its size is $3 \log p$ bits).

A multiplication by $A$ or $B$ in $S L_{2}\left(F_{p}\right)$ requires essentially 4 additions, so hashing an $n$ bit text requires $4 n$ additions of $\log p$ bits, which is reasonably fast. Signatures based on associating an alphabet with basic matrices and multiplying them in $G L_{2}\left(F_{p}\right)$ have been proposed before, but with basic matrices of arbitrary size, so that a large $p$ could not be chosen without damaging the speed of computation, and forging could be achieved with probabilistic methods of factoring, see [Cam], which do not seem to be appliable here.

The problem of devising a forgery involves factoring elements of $S L_{2}\left(F_{p}\right)$ into a product of $A$ 's and $B$ 's. Trivial factorisations of an arbitrary matrix of $S L_{2}\left(F_{p}\right)$ can be exhibited but they have a length in $O(p)$, so are useless as a forgery (no text has $2^{150}$ bits !). What is needed is a short factorisation of elements of $S L_{2}\left(F_{p}\right)$ which seems to be quite difficult, see [Bab] ; besides, recall that the general problem of finding the shortest factorisation of an arbitrary element of a group over a given set of generators is P-space complete, (see [Jer]).

"Local" modifications of the text change the signature : Suppose that a subset of $k$ consecutive bits $x_{1} x_{2} \ldots x_{k}$ of a text is changed into $h$ consecutive bits $x_{1}^{\prime} x_{2}^{\prime} \ldots x_{h}^{\prime}$, with $x_{1} \neq x_{1}^{\prime}$ and $x_{k} \neq x_{h}^{\prime}$. If the signature is unchanged, then the corresponding products of $A$ 's and $B$ 's are equal in $S L_{2}\left(F_{p}\right)$ :

$$
X_{1} X_{2} \ldots X_{k}=X_{1}^{\prime} X_{2}^{\prime} \ldots X_{h}^{\prime} \quad \bmod p
$$

Evaluate those two products in $S L_{2}(Z)$; they are necessarily different : to see this, recall that $S L_{2}(Z)$ is classically generated by the matrices

$$
S=A \text { and } T=\left(\begin{array}{rr}
0 & 1 \\
-1 & 0
\end{array}\right)=-S^{-1} B S^{-1}=\left(S^{-1} B S^{-1}\right)^{3}
$$


Proposition 3 The diameter of the directed Cayley graph $\mathcal{C}\left(S L_{2}\left(F_{p}\right), A, B\right)$ is in $O(\log p)$.

The proof involves powerful (and nonconstructive) arithmetic : it can be shown (see [Bab] and the references therein) that there is a constant $c$ (independant of $p$ ) such that for any set $X$ of vertices of the nondirected Cayley graph $\mathcal{C}\left(G=P S L_{2}\left(F_{p}\right), A, B, A^{-1}, B^{-1}\right)$, such that $|X| \leq \frac{1}{2}|G|$

$$
|\partial X| \geq c|X|
$$

where $\partial X$ denotes the set of neighbours of $S$, not in $X$. Following [AlM] it can be deduced that the diameter of $\mathcal{C}\left(P S L_{2}\left(F_{p}\right), A, B, A^{-1}, B^{-1}\right)$ is in $O(\log p)$.

We can adapt those combinatorial techniques to obtain (4) in the directed graph case and hence proposition 3.

Generalisations to strings over larger alphabets can be envisaged, using matrices that generate graphs with a large girth and a small diameter.

\section{References}

[AlM.85] N. Alon, and V.D. Milman, " $\lambda_{1}$, isoperimetric inequalities for graphs, and superconcentrators", J. Comb. Theory. Ser. B, 38 (1985), 73-88.

[Bab.89] L. Babai, W.M. Kantor, and A. Lubotsky, "Small-diameter Cayley Graphs for Finite Simple Groups," Europ. J. Combinatorics, 1989, $10,507-522$.

[Cam.87] P. Camion, "Can a Fast Signature Scheme Without Secret Key be Secure ?", in AAECC, Lecture Notes in Computer Science, n 228, Springer-Verlag.

[Jer.85] M.R. Jerrum, "The Complexity of Finding Minimum Length Generator Sequences", Theoretical Computer Science, April 1985.

[God.88] P. Godlewski and P. Canion, "Manipulations and Errors, Detection and Localization", Advances in Cryptology, EUROCRYPT-88, Springer-Verlag, pp.96-106.

[Mar.82] G.A. Margulis, "Explicit Constructions of Graphs Without Short Cycles and Low Density Codes," COMBINATORICA 2 (1), 1982, 71-78. 